\title{
Some Approaches to Symmetric Spaces
}

\author{
M-Alamin A. H. Ahmed \\ Departmemt of mathematics , Faculty of Science and Arts - Khulais , King Abdulaziz University, Saudi Arabia
}

\begin{abstract}
In this study we introduce some approaches, geometrical and algebraic which help to give further understanding of symmetric spaces and help scientists who are seeking for suitable spaces for their applications to carry on their job. One of the aims of this study is to put forward the close connection between different approaches to symmetric spaces, namely algebraic and geometrical features of these spaces with some results. Also giving some conclusions and remarks on the foundation of symmetric spaces with logical ordering of notions and consequences. We think that symmetric space is a very important field for understanding abstract and applied features of spaces, besides they are still need much effort to understand them because of the diversity of their approaches which mix between algebra and geometry, so this Paper is an attempt to disclose some of these features and helps in more understanding and put forward a base for future applications .
\end{abstract}

Keywords Riemannian Manifold, Lie Groups, Lie Algebras, Homogeneous Spaces, Curvature Tensor

\section{Introduction}

In studying spaces, one of the aims of this study is to introduce spaces that can suit some scientific applications . Many scientific problems in various fields may have their own conditions that might not agree with the geometric structure and properties of some spaces familiar to mathematicians and geometers .

Historically the Euclidean space, then the Cartesian and so on have been serving the scientific needs and the solutions to many problems, but mathematicians always think of new spaces whenever it is crucial to do that, or also they introduce new spaces to be ready for future applications .

In differential geometry, manifolds do their role to meet some scientific demands. This is due to some properties of man ifolds, for instance manifolds need not be connected, or closed. They are never countable unless their dimension is zero .

Differentiable manifolds for example lead to Lie groups, and these with their Lie algebras help to introduce some abstract spaces such as symmetric spaces .

When we introduce symmetric spaces, this can be done through different approaches of algebraic and geometric nature.

In this study we are going to discuss some of these approaches which lead to some invariant properties of symmetric spaces. We can look to these spaces from different aspects which unify some algebraic and geometric

* Corresponding author:

modhsd@gmail.com (M-Alamin A. H. Ahmed)

Published online at http:/journal.sapub.org/ajms

Copyright (C) 2012 Scientific \& Academic Publishing. All Rights Reserved properties .

\section{Preliminaries}

\subsection{Smooth Manifold}

Manifolds are topological spaces that are locally Euclidean and Hausdorff.

A smooth manifold $M$ is a manifold endowed with a smooth structure, that is an atlas of charts satisfying smoothness conditions .

Simple examples of smooth manifolds are the Euclidean n-space $\mathbb{R}^{n}$ and unit sphere $S^{n}$.

\subsection{Proposition}

A manifold is locally connected, locally compact, a union of a countable collection of compact sets, normal and meterizable .

\subsection{A Smooth Tensor Field}

Let $M$ be a smooth manifold. A s mooth tensor field $A$ on the manifold $M$ of type $(r, s)$ is the map $A: C_{r}^{\infty}(T M) \rightarrow C_{s}^{\infty}(T M)$, which is multi- linear over $C^{\infty}(M)$, that is, it satis fies the following :

$$
\begin{aligned}
& A\left(X_{1} \otimes \ldots \otimes X_{k-1} \otimes(f . y+h . Z) \otimes X_{k+1} \otimes \ldots . \otimes X_{r}\right)= \\
& f . A\left(X_{1} \otimes \ldots . \otimes X_{k-1} \otimes y \otimes X_{k+1} \otimes \ldots . \otimes X_{r}\right)+ \\
& h . A\left(X_{1} \otimes \ldots . \otimes x_{k-1} \otimes Z \otimes X_{k+1} \otimes \ldots . \otimes X_{r}\right), \\
& \forall X_{1}, \ldots ., X_{r}, Y, Z \in C^{\infty}(T M), \\
& f, h \in C^{\infty}(T M) \text { and } k=1, \ldots ., r
\end{aligned}
$$


Shortly for the s mooth tensor field $A\left(X_{1}, \ldots \ldots, X_{r}\right)$, We use the notation

$A\left(X_{1}, \ldots \ldots, X_{r}\right)$. Also $T M$ denotes the tangent bundle on $M$, that is the disjo int union of all tangent spaces on $M$.

\subsection{Proposition}

Let $A: C_{r}^{\infty}(T M) \rightarrow C_{s}^{\infty}(T M)$ be a tensor field of type $(r, s)$ and the point $p \in M$. Let $X_{1}, \ldots, X_{r}$ and $Y_{1}, \ldots ., Y_{r}$ be smooth vector fields on $M$ such that $\left(X_{k}\right)_{p}=\left(Y_{k}\right)_{p}$ for each $k=1, \ldots ., r$, then

$$
A\left(X_{1}, \ldots \ldots, X_{r}\right)(p)=A\left(Y_{1}, \ldots . ., Y_{r}\right)(p)
$$

\section{5. Definition ( Riemannian Metric )}

A Riemannian metric $g$ on a manifold $M$ is a tensor field

$g: C_{2}^{\infty}(T M) \rightarrow C_{0}^{\infty}(T M)$ such that for each $p \in M \quad$ the restriction $\quad g_{P}=g$ $T_{p} M \otimes T_{p} M: T_{p} M \otimes T_{p} M \rightarrow \mathbb{R} \quad$ with $\quad g_{P} \quad:$ $\left(X_{p}, Y_{p}\right) \rightarrow g(X, Y)(p)$ is an inner product on the Tangent space $T_{p} M$.

\subsection{Connections and Riemannian Connections}

In a s mooth $\mathrm{n}-$ man ifold $M$ with a smooth Riemannian metric $g$, let $V$ be the set of all smooth vector fields on $M$. A connection on $M$ is the operator $\nabla(X, Y)=\nabla_{X} Y$, satisfying the conditions :

$$
\begin{aligned}
& i-\nabla_{X_{1}+X_{2}} Y=\nabla_{X_{1}} Y+\nabla_{X_{2}} Y \\
& i i-\nabla_{X}\left(Y_{1}+Y_{2}\right)=\nabla_{X} Y_{1}+\nabla_{X} Y_{2} \\
& i i i-\nabla_{f x} Y=f \nabla_{X} Y, \\
& f: M \rightarrow \mathbb{R} \text { is a smooth function } \\
& i v-\nabla_{X}(f Y)=X(f) Y+f \nabla_{X} Y
\end{aligned}
$$

If in addition to the above conditions we have

$$
\begin{aligned}
& v-X(g(Y, Z))=g\left(\nabla_{X} Y, Z\right)+g\left(Y, \nabla_{X} Z\right), \\
& \forall X, Y, Z \in V v i-\nabla_{X} Y-\nabla_{Y} X=[X, Y],
\end{aligned}
$$

the connection $\nabla$ is called the Riemannian connection .

We can use for a chart $(U, \phi)$ on n- manifold :

$$
\begin{gathered}
\nabla_{i} Y:=\nabla_{\frac{\partial}{\partial x^{i}}} Y, \text { then } \\
\nabla_{i} \frac{\partial}{\partial x^{i}}=\sum_{k} \Gamma_{i j}^{k} \frac{\partial}{\partial x^{k}}, \\
\Gamma_{i j, k}=g\left(\nabla_{i} \frac{\partial}{\partial x^{j}}, \frac{\partial}{\partial x^{k}}\right)
\end{gathered}
$$

(The Chistoffel symbols) .

If $X=\sum a^{i} \frac{\partial}{\partial x^{i}}, Y=\sum b^{i} \frac{\partial}{\partial x^{j}}$, then using (vi) above we find the covariant derivative ( or the connection ):

$$
\nabla_{X} Y=\sum_{k}\left[\sum_{i} a^{i} \frac{\partial b^{k}}{\partial x^{i}}+\sum_{i, j} a^{i} b^{j} \Gamma_{i j}^{k}\right] \frac{\partial}{\partial x^{k}}
$$

\subsection{Par allel Vector Fields \& Geodesics}

If $M$ is a smooth $\mathrm{n}$ - manifold with a smooth Riemannian metric $g$.

Let $\nabla$ be the unique Riemannian connection on $M$ corresponding to $g$. Let $C(t)$ be a smooth regular curve in $M, Y(t)$ be a vector field along $C$. Then $Y$ is parallel along $C$ if $\nabla_{\partial / \partial t} Y=0$ and $C$ is a geodesic in $M$ if $\partial C / \partial t$ is parallel along $C$, that is, if $\left(\nabla_{\partial C / \partial t}\right)(\partial C / \partial t)=0$

\subsection{Rie manni an Manifold}

Let $M$ be a smooth manifold with a metric $g$, the pair ( $M, g$ ) is called a Riemannian manifold. Geometric properties of ( $M, g$ ) which only depend on the metric $g$ are called intrinisic or metric properties.

\subsection{Levi - Civita Connection}

In a Riemannian manifold with a metric $g$, a connection $\nabla$ is called a Levi - Civita connection if it is torsion- free ( $T(X, Y)=0$ ), and is compatible with $g$, Where :

$$
\begin{aligned}
& 2\left\langle\nabla_{X} Y, Z\right\rangle=X(\langle Y, Z\rangle)-Z(\langle Y, X\rangle)+Y(\langle Z, Y\rangle) \\
& -\langle Z,[Y, X]\rangle+\langle[Z, Y], X\rangle-\langle Y,[X, Z]\rangle .
\end{aligned}
$$

\section{Lie Groups and Lie Algebras}


Lie groups and their Lie algebras are very useful and important tools when studying symmetric spaces, this results from the fact that their algebraic properties derive from the group axioms, and their geometric properties derive from the identification of group operation $\mathrm{s}$ with points in a topological spaces, and these are man ifo lds .

We should note that every Lie group is a smooth man ifold .

\subsection{Definition( Lie Group)}

A Lie group $G$ is a group satisfying two additional axio $\mathrm{ms}$ :

(i) The mapping of the group operation $G \times G \rightarrow G$ defined by $(x, y) \rightarrow x y$, and

(ii) The inverse map $G \rightarrow G$ defined by $x \rightarrow x^{-1}$, are both smooth .

Examples:

$G L(n, \mathbb{R}) \& O(n)$ are examples of Lie groups .

\subsection{Definition ( Lie Algebra of a Lie Group )}

The tangent space to a linear Lie group $G$ at the identity $e$, denoted $\mathrm{g}=T_{e} G$ is its Lie algebra endowed with a ( non-associative ) multip lication, the Lie bracket satisfying the axioms of a Lie algebra as a vector space .

\subsection{Homomor phisms of Lie Groups and Lie Algebras}

The homomorphism of the Lie groups $G_{1}$ and $G_{2}$ is a function $f: G_{1} \rightarrow G_{2}$ which is a homomorphism of groups and a smooth map between the manifolds $G_{1}$ and $G_{2}$.

If $g_{1}$ and $g_{2}$ are two Lie algebras, also the homomo rphism of Lie algebras is a function $f: g_{1} \rightarrow g_{2}$ which is a linear map between the vector spaces $\mathrm{g}_{1}$ and $\mathrm{g}_{2}$ preserving Lie brackets, that is $f[X, Y]=[f(X), f(Y)]$.

\subsection{Left and Right Translations}

If $G$ is a Lie group, for every $a \in G$ we define :

(i ) Left translation as the map $L_{a}: G \rightarrow G$ such that $L_{a}(b)=a b, \forall b \in G$.

(ii ) Right translation as $R_{a}: G \rightarrow G$ such that $R_{a}(b)=b a, \forall a, b \in G \quad . \quad L_{a} \& R_{a} \quad$ are both diffeomorphisms , $R_{a^{-1}} L_{a}$ or $\mathrm{Ad}_{a}$ is given by $R_{a^{-1}} L_{a}(b)=a b a^{-1}$
The derivative $d\left(R_{a^{-1}} L_{a}\right)_{e}: \mathrm{g} \rightarrow \mathrm{g}$ is an isomorphism of Lie algebras, denoted by $\mathrm{Ad}_{a}: \mathrm{g} \rightarrow \mathrm{g}$.

That is, $d\left(R_{a^{-1}} L_{a}\right)_{e} \equiv \operatorname{Ad}_{a}$.

\subsection{Adjoint Representation}

If we denote the Lie group of all bijective linear maps on $\mathrm{g}$ by $\mathrm{GL}(\mathrm{g})$, then we call the map $a \rightarrow A d_{a}$ where $A d: G \rightarrow G L(\mathrm{~g})$ the adjo int representation of $G$.

g. If $G$ is a linear group we have $A d(a)(X)=A d_{a}(X)=a X a^{-1}, \forall a \in G \quad \& \forall X \in$ The derivative $d A d_{e}: \mathrm{g} \rightarrow \mathrm{gL}(\mathrm{g})$ denoted by $a d: \mathrm{g}$ $\rightarrow \mathrm{gL}(\mathrm{g})$ is called the adjoint representation of $\mathrm{g}$ ( where $\mathrm{gL}(\mathrm{g})$ denotes the Lie algebra, End $(\mathrm{g}, \mathrm{g})$ of all linear maps on $g$ ).

In the case of linear group we have ad $(X)(Y)=[X, Y], \forall X, Y \in \mathrm{g}$, and

$$
\operatorname{ad}([X, Y])=[\operatorname{ad}(X), \operatorname{ad}(Y)], \forall X, Y \in
$$

$\mathrm{g}$. According to what is mentioned we can define the Lie algebra of the Lie group as :

\subsection{Defini tion ( Lie Bracket)}

Given a Lie group $G, \mathrm{~g}=T_{e} G$ with the Lie bracket defined by : $[X, Y]=a d(X)(Y), \forall X, Y \in \mathrm{g}$, is the Lie algebra of the Lie group $\mathrm{G}$.

\subsection{Left and Right Invariant Vector Fields}

Given $G$ is a Lie group, we define a vector field $\xi$ on $G$ as :

(i) Left-invariant vector field if and only if

$d\left(L_{a}\right)_{b}(\xi(b))=\xi\left(L_{a}(b)\right)=\xi(a b), \forall a, b \in G$ , and

(i ) Right - invariant vector field if and only if $d\left(R_{a}\right)_{b}(\xi(b))=\xi\left(R_{a}(b)\right)=\xi(b a), \forall a, b \in G$.

If $\xi$ is a left - invariant vector field, putting $b=e$, we have :

$\xi(a)=d\left(L_{a}\right)_{e}(\xi(e))$, that is $\xi$ is determined by its value $\xi(e) \in \mathrm{g}$, at the identity $e$.

The map $\xi \rightarrow \xi(e)$, establishes an isomorphism between the space of left - invariant vector fields on $G$ and g. 
In fact for $X \in \mathrm{g}$, we define the vector field $X^{L}(a)=d\left(L_{a}\right)_{e}(X), \forall a \in G$, then the map $G \times \mathrm{g} \rightarrow T G$ given by $(a, X) \rightarrow X^{L}(a)$ is an isomorphis $\mathrm{m}$ between $G \times \mathrm{g}$ and the tangent bundle $T G$.

\subsection{Definition ( Lie Subalgebra and the Ideal )}

If $\mathrm{g}$ is a Lie algebra, a subalgebra $\eta$ of $\mathrm{g}$ is a subspace of $\mathrm{g}$ such that $[u, v] \in \eta, \forall u, v \in \eta$. If $\eta$ is a subspace of $\mathrm{g}$ such that $[u, v] \in \eta, \forall u \in \eta$ and all $v \in \mathrm{g}$, we call $\eta$ an ideal in $\mathrm{g}$.

Note that many properties of Lie groups structure can be studied and derived through their Lie algebras, that is why they are important to be studied .

A simple Lie algebra has no proper ideal. The semisimple algebras are constructed of simple ones .

\subsection{The Exponential Map}

In a Riemannian manifold $(M, \mathrm{~g})$ with the point $p \in M$, the exponential map $\exp _{p}: T_{P} M \rightarrow M$ is defined by $\quad \exp _{p}:(V):=\gamma_{v}(e) \quad$ where $\gamma_{v}:[0, \infty] \rightarrow M$ is the constant speed geodesic emanating from $p$ with $\gamma_{v}(0)=V$ and $\gamma_{v}(t)=\exp _{p}(t v)$.

The Lie algebra $g$ generates a group through the exponential mapping. A general group element is $M=\exp \left(\sum_{i} t^{i} X_{i}\right), t_{i} \in F, X_{i} \in \mathrm{g}$.

If we choose a basis $\left[X_{i}\right]$ for $\mathrm{g}$. Then $a d X_{i}\left(X_{j}\right)=\left[X_{i}, X_{j}\right]=C_{i j}^{k} X_{k}$, we sum over k. The $C_{i j}^{k}$ are realstructure constants .

The structure constants define the matrices $M$ of the adjoint representation through $\left(M_{i}\right)_{j k}=C_{i k}^{j}$.

\subsection{Cartan Subalgebra}

In a simple Lie algebra, we have two kinds of generators :

(i) The Cartan subalgebra, which is a maximal abelian subagebra $H_{0}=\left\{H_{1}, \ldots . ., H_{r}\right\}$ such that $\left[H_{i}, H_{j}\right]=0$.

On representing each of the Lie algebra by $n \times n$ matrix, then $\left[H_{i}, H_{j}\right]=0$ means the matrices $H_{i}$ can all be diagonalized simultaneously .
Their eigenvalues $\mu_{i}$ are given by $H_{i}|\mu\rangle=\mu_{i}|\mu\rangle$, where the eigenvectors are labeled by the weight vectors $\mu=\left(\mu_{i}, \ldots . ., \mu_{r}\right)$.

(ii) There are raising and lowering operators denoted $E_{\alpha}$ such that

$$
\left[H_{i}, E_{\alpha}\right]=\alpha_{i} E_{\alpha},\left[E_{\alpha}, E_{-\alpha}\right]=\alpha_{i} H_{i} \text {. }
$$

$\alpha$ is an $\mathrm{r}$ - dimensional vector called a root, $\alpha=\left(\alpha_{1}, \ldots \ldots, \alpha_{r}\right)$ and $\mathrm{r}$ is the rank of the algebra .

The roots form a lattice in the space dual to the Cartan subalgebra. They are useful in the problem of classification of symmetric spaces.

\subsection{Killing For m}

If $\mathrm{g}$ is a Lie algebra, we define the Killing form $B$ of $\mathrm{g}$ over afield $F$ as the bilinear form :

$$
B: \mathrm{g} \times \mathrm{g} \rightarrow F,(X, Y) \rightarrow \operatorname{tr}(\operatorname{ad} X \circ a d Y) .
$$

The Lie group and its Lie algebra are called semisimple if the Killing form is nondegenerate .

\section{Homogeneous Space}

To introduce homogeneous spaces we should remember the action of the group on a set .

\subsection{The G-s pace}

A locally compact Hausdorff space equipped with a transitive action of the group $G$, is called a $G$-space .

\subsection{The ore $\mathrm{m}$}

Let $G$ be a locally compact group and let $X$ be a transitive $G$-space. Let $x_{0} \in X$ and $H$ be the isotropy group of $x_{0}$. If $G$ is compact, then $\Phi: G / H \rightarrow X$ given by $g H \rightarrow g \cdot x_{0}$ is a homomorphis $\mathrm{m}$.

\subsection{Defini tion ( Homogene ous S pace )}

A Homogeneous space $X$ is a transitive $\mathrm{G}-$ space that is isomorphic to a quotient space $G / H$. That is, there is an isomorphis $\mathrm{m}$ by the mapping $\Phi$ as in the previous theorem, making $G / H$ isomorphic to $X$.

In a homogeneous space every point looks exactly like every other point. We can also look at a homogeneous space as a space whose is ometry group acts transitively on it .

\subsection{Example}

As an example of a homogeneous space, the n-sphere $S^{n}=\left\{x \in \mathbb{R}^{n+1}=\|x\|=1\right\}$, its isometry group is the 
group of rotations given by $S O(n+1)$. For every two points $x, y \in S^{n}, \exists \sigma \in S O(n+1) \mid \sigma x=y$, so that there is a transitive action and $S^{n}$ is a homogeneous space .

\section{Symmetric Spaces}

These are spaces which possess the properties of symmetry and homogeneousness, and they have many applications ,this is because they have mixed algebraic and geometric properties. The beginning for these spaces is that they are spaces with parallel curvature tensor, later they were introduced through different approaches. They have much in common. Any symmetric space has its own special geometry, Euclidean, elliptic and hyperbolic are some of these geometries.

We give some approaches to symmetric spaces using some algebraic and geometric properties.

\subsection{Some Approaches to Symmetric S paces}

A symmetric space can be considered as :

1 - A Riemannian manifold with point reflection .

2 - A Riemannian manifold with parallel curvature tensor .

3 - A Lie group with certain involution .

4 - A homogeneous space with special isotropy group .

5 - A Riemannian manifold with special holonomy .

6 - A special Killing vector field .

7 - A Lie triple system.

These may be some of many other approaches to symmetric spaces, but we are interested in this work in some of these approaches to reach the required aims of this study .

\section{Symmetric Spaces and Parallel Curvature Tensor}

The most famous approach to symmetric spaces is that related to Ellie Cartan, we introduce it here to complete the picture of our opinions :

\subsection{Definition}

A Riemannian manifold $M$ is called locally symmetric if its curvature tensor $R$ is parallel, that is $\nabla R=0$.

\subsection{The ore m ( E. Cartan )}

Let $M$ be a Riemannian manifold. Then $M$ is locally symmetric $\Leftrightarrow \nabla R=0$, where $R$ is the curvature tensor of $M$, and $\nabla$ is the connection induced on 4-tensor on $M$ by the Levi-Civita connection of $M$.

\subsection{The ore m (Symmetric Space )}

A Complete, Locally symmetric, simply connected Riemannian manifold is a symmetric space .
According to what is mentiond above we can give many examples of symmetric spaces, where we shall give some details on some of them when introducing other approaches to symmetric spaces. Briefly we can say that $E^{n}$ ( the Euclidean space), $S^{n}$ ( the unit sphere in $\mathbb{R}^{n+1}$ ) and $H^{n}$ (the hyperbolic space ) are all examp les of symmetric spaces.

\section{Symmetric Spaces with Point Reflection}

Here we consider symmetric spaces as Riemannian manifolds with point reflections :

Let $M$ be a Riemannian manifold, $P$ a point of $M$. If the geodesic reflection at any point $p \in M$ is an isometry of $M$, that is $S_{P} \in G=I(M)$, the isometry group of $M$, such that

$s_{P}(P)=P \quad,\left(d s_{P}\right)_{P}=-I$.

The isometry $S_{P}$ is called symmetry at $P$. From this definition, $M$ is geodesically complete and homogeneous .

We can say that a Symmetric space $M$ is precisely a homogeneous space with a symmetry $S_{P}$ at some point $p \in M$.

We identify the homogeneous space $M$ with the coset space $G / K$ where $G$ is the isometry group of $M$, and $K$ is the isotropy group $G_{P}=\{g \in G: g(P)=P\}$.

\subsection{Definition ( Locally Symmetric Space )}

A locally symmetric space is a Riemannian manifold in which the geodesic symmetry at each point is an isommetry in a normal neighbourhood of the point. Symmetric Spaces are locally symmetric too, the geodesic symmetries in this case are global is o metries .

\subsection{Example ( the Unit Sphere )}

Let $S^{n}$ be the unit sphere in $\mathbb{R}^{n+1}$ with the standard scalar product. On taking the symmetry at any point $P \in S^{n}$ as the reflection at the line $L_{P}$ in $\mathbb{R}^{n+1}$, that is $S_{P}(y)=-y+2\langle y, x\rangle x$. This symmetries generate the full isometry group which is the orthogonal group $O(n+1)$. The isotropy group of the last standard unit vector $e_{n+1}=(0,0, \ldots \ldots, 0,1)^{T}$ is $O(n) \subset O(n+1)$.

\subsection{Example ( the Or thogonal Group ) (Abstract)}


Let $\quad M=O(n)=\left\{g \in \mathbb{R}^{n \times n}: g^{T} g=I\right\} \quad, \quad$ a

Submanifold of the matrix space $\mathbb{R}^{n \times n}$.

The Riemannian metric on $O(n)$ is induced from the trace scalar product on $\mathbb{R}^{n \times n}$ :

$\langle x, y\rangle:=$ trace $x^{T} y=\sum_{i j} x_{i j} y_{i j}$.

For any $g \in O(n)$ and $x, y \in \mathbb{R}^{n \times n}$ we have :

$\langle g x, g y\rangle=\operatorname{trace}\left(x^{T} g^{T} g y\right)$

$=\operatorname{trace} x^{T} y=\langle x, y\rangle$

$\langle x g, y g\rangle=\operatorname{trace}\left(g^{T} x^{T} y g\right)$

$=\operatorname{trace}\left(g^{-1} x^{T} y g\right)=\operatorname{trace} x^{T} y=\langle x, y\rangle$

( the inner product is preserved). Right and left multiplications with $g \in O(n)$ preserve the subset $O(n) \subset \mathbb{R}^{n \times n}$, so they act as iso metry on $O(n)$ turning $O(n)$ into a homogeneous space.

The linear map $S_{I}$ on $\mathbb{R}^{n \times n}$ given by $s_{I}(x)=x^{T}$ is an isometry of $O(n)$ preserving $O(n) \subset \mathbb{R}^{n \times n}$ and the scalar product of $\mathbb{R}^{n \times n}$. This is the symmetry at the identity matrix $I \in O(n)$ since it fixes $I$ and acts as $-I$ on the tangent space $T_{I}(O)=\left\{v \in \mathbb{R}^{n \times n}: v^{T}=-v\right\}$. The symmetry at an arbitrary element $g \in O(n)$ is given by $s_{g}(x)=g\left(s_{I}\left(g^{T} x\right)\right)=g x^{T} g \quad, \quad$ noting that $s_{g}(g)=g$ and for any $g v \in T_{g} O(n)=g_{I}^{T} O(n) \quad, \quad$ we have $d s_{g}(g v)=s_{g}(g v)=g v^{T}=-g v$.

\section{Symmetric Space as a Lie Group with Involutive Isometry}

This approach can be seen through point reflection, then we can introduce the notion of involution and symmetric pairs with involutive automorphis $m$.

\subsection{Definition ( Symmetric S pace and Involution )}

Let $M$ be a Riemannian manifold with the metric $g$ such that for every point $P \in M$ there exists an isometry $\sigma_{P}$ of $(M, g)$ called an involution such that :

(1) $\sigma_{P}(P)=P \quad$ and

$$
\text { (2) } d \sigma_{P}=-i d T_{P} M
$$

Composition of involutions will get translations along geodesics which can be used to extend geodesics to the whole of $\mathbb{R}$ and this means $M$ is geodesically complete.

\subsection{The Role of Lie Groups}

Again we consider Lie groups in this approach, this is through the fact that the group of isometries mentioned above is a Lie group .

By Hopf - Rinow theorem any two points in a geodesically complete Riemannian manifold can be connected by a geodesic .

We deduce that the translations along the geodesics make the isometry group $G$ ( Lie group ) acting on $M$ transitively. Here also we identify $M$ with $G / K$ (Homogeneous space), where $K$ is the is otropy group at a point $P \in M$.

\section{Characterization of Symmetric Spaces}

One of the most surprising features of symmetric spaces is that we can get their properties and information about them from their groups of isometry and this is another scope for more study .

\subsection{The Triple $(\mathrm{g}, \boldsymbol{\eta}, \rho)$}

In the symmetric space $M$, let $G$ be its group of isometry and $H$ is the isotropy group at $P \in M$ then $G$ has a Lie group structure and $H$ is a closed Lie subgroup of $G$. The Lie algebra $g$ of $G$ is the space of Killing vector fields on $M$. The Lie algebra $\eta$ of $H$ is a subalgebra of $g$ and has a natural complementary subspace $\rho$ such that :

$\mathrm{g}=\eta \oplus \rho,[\eta, \eta] \subset \eta,[\eta, \rho] \subset \rho$ and $[\rho, \rho] \subset \rho$.

So the characterization of symmetric spaces is the same as characterization of such triples

$(\mathrm{g}, \eta, \rho)$.

\section{Results and Conclusions}

$\rightarrow$ The geometric and algebraic approaches to symmetric spaces can be modified to deduce each other.

$\rightarrow$ Most of features of symmetric spaces can be extracted fro $m$ their Lie algebras or the triple $(g, \eta, \rho)$. 
$\rightarrow$ The study of symmetric spaces and continuous research in their properties and classification can lead to most surprising results that can help in their applications .

-The different approaches to symmetric spaces mentioned in this paper, lead to specifying two important features of symmetric spaces, that is homogeneousness and symmetry .

Homogeneousness can be considered as algebraic property through the transitive action of the isometry group while symmetry is a geometric property which can be seen through point reflection in the Riemannian manifold .

An important remark on symmetric spaces is that we can use them in describing some abstract and non- abstract spaces, and this unifies the theory and application of spaces which pave the way for future applications in many fields .

\section{REFERENCES}

[1] S. Helgason : Differential Geometry , Lie Groups and Symmetric Spaces , Academic Press 1978.

[2] R. S . Palais , C. - L. Terng : Critical Point Theory and Submanifold Geometry, Springer L . N . in math. 1353 , 1988 .

[3] O .Loos : Symmetric Spaces I, II , Benjamin 1969 .

[4] B . O' Neill, Semi Riemannian Geometry , Academic Press (1983)

[5] J . M . Lee . Riemannian Geometry, an introduction to curvature. Springer 1997 .

[6] S . Kobayashi, K. Nomizu : Foundations of Differential geometry , I, II, Wiley 1973 .

[7] J . M . Lee . Introduction to smooth Manifolds, Springer, 2006 . 